\title{
CORRIGENDUM
}

\section{Timing of cyclin D1 expression within G1 phase is controlled by Rho}

\author{
Catherine F. Welsh, Kristin Roovers, Jessie Villanueva, YunQi Liu, Martin A. Schwartz and Richard K. Assoian
}

Nature Cell Biol. 3, 950-957 (2001); published online 5 October 2001

In the version of the article initially published, two panels contain duplicated bands. The left hand side part of the Cdk4 northern blot in Fig. 2c is identical to the G0 panel of the ERK1/ERK2 western blot. The northern blot data with the correct data is displayed below. The vector control panel of the ERK1/2 western blot of Fig. 3a contains one lane which was duplicated and spliced in an inverted form. The original underlying data was not found, but unmanipulated data from alternative experiments allowing the same conclusions are presented below. In Fig. $1 \mathrm{c}$ the $6 \mathrm{~h}$ timepoint should have been labelled $3 \mathrm{~h}$ (although the excised $6 \mathrm{~h}$ timepoint is quantitatively similar). In the northern blots presented in Fig. 1c, there is a splice between lanes 3 and 4 of both blots, which should have been marked; we confirm that the samples were run

Figure 2c

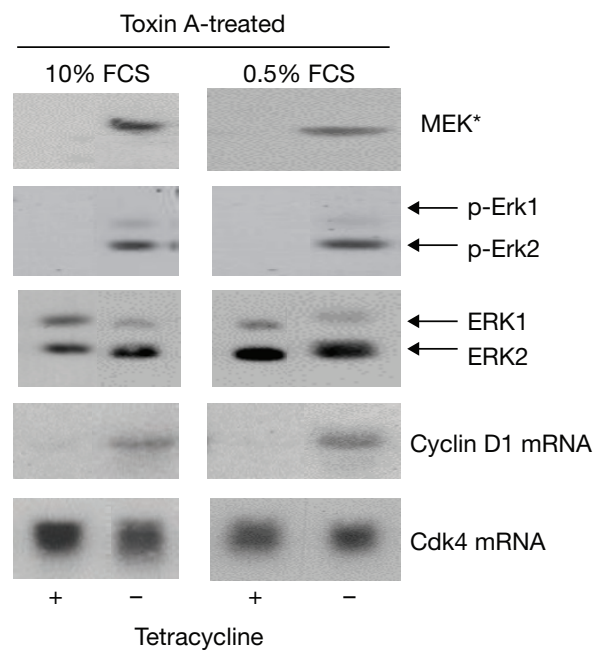

Figure 4b

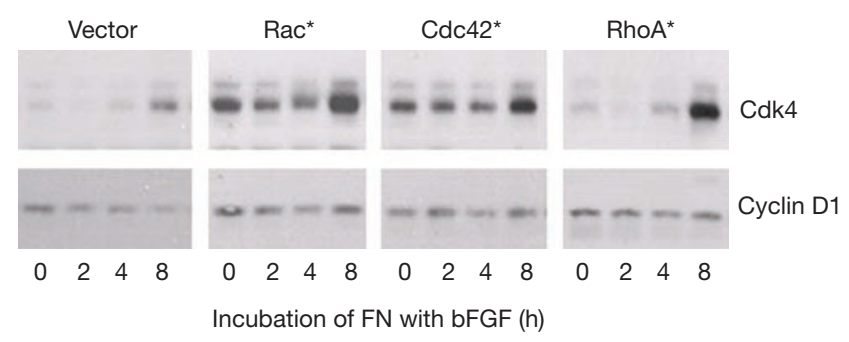

on the same gel. In Fig. 4b, a darker exposure of the $8 \mathrm{~h}$ lane was inserted in the vector blots for cyclin D1 and cdk4. Below is the unmanipulated data. The conclusions are unaltered. In Fig. $5 \mathrm{~b}$ there is a splice between lanes 5 and 6 of both blots, which should have been marked; we confirm that the samples were run on the same gel. In Fig. 6a, a lighter exposure was inserted at $4.5 \mathrm{~h}$ in both the GTP-Rho and Total Rho panels. Below is the unmanipulated data. The conclusions are unaltered. We apologize for these serious mistakes, and note that the conclusions of the paper stand. Finally, it should be noted that samples from a single experiment were fractionated on different gels to assess ERK activity by gel-shift and by phosphoERK levels as indicated in the Methods. This precludes direct quantitative comparisons between the panels.

Figure 3a

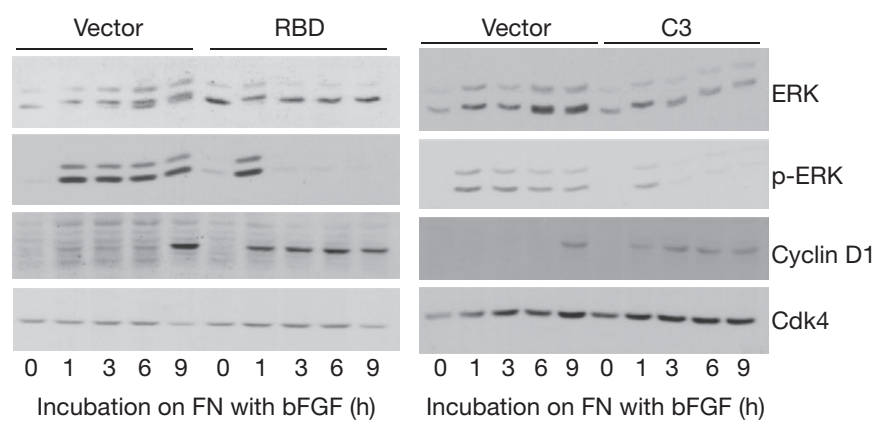

Figure 6a

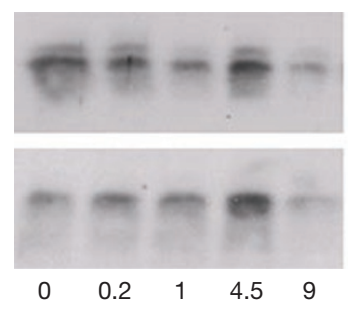

Controls

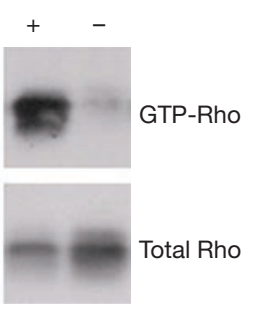

Incubation on FN with bFGF (h) 